\title{
The interpeduncular fossa approach for resection of ventromedial midbrain lesions
}

\author{
${ }^{*}$ M. Yashar S. Kalani, MD, PhD, Kaan Yağmurlu, MD, and Robert F. Spetzler, MD \\ Department of Neurosurgery, Barrow Neurological Institute, St. Joseph's Hospital and Medical Center, Phoenix, Arizona
}

The authors describe the interpeduncular fossa safe entry zone as a route for resection of ventromedial midbrain lesions. To illustrate the utility of this novel safe entry zone, the authors provide clinical data from 2 patients who underwent contralateral orbitozygomatic transinterpeduncular fossa approaches to deep cavernous malformations located medial to the oculomotor nerve (cranial nerve [CN] III). These cases are supplemented by anatomical information from 6 formalin-fixed adult human brainstems and 4 silicone-injected adult human cadaveric heads on which the fiber dissection technique was used.

The interpeduncular fossa may be incised to resect anteriorly located lesions that are medial to the oculomotor nerve and can serve as an alternative to the anterior mesencephalic safe entry zone (i.e., perioculomotor safe entry zone) for resection of ventromedial midbrain lesions. The interpeduncular fossa safe entry zone is best approached using a modified orbitozygomatic craniotomy and uses the space between the mammillary bodies and the top of the basilar artery to gain access to ventromedial lesions located in the ventral mesencephalon and medial to the oculomotor nerve.

https://thejns.org/doi/abs/10.3171/2016.9.JNS161680

KEY WORDS brainstem surgery; interpeduncular fossa; mesencephalon; safe entry zone; surgical technique; ventromedial midbrain

$\mathrm{T}$ HE human brainstem serves as a relay center for ascending and descending fiber tracts that are essential for motor and sensory control. The densely packed nature of eloquent tracts and nuclei in the brainstem has historically caused great trepidation when surgeons approach lesions that do not abut an ependymal or a pial surface. More recently, surgical innovation and development of novel tools have enabled some neurosurgeons to approach deep-seated lesions using several safe entry zones-areas where the brainstem may be traversed with minimal or acceptable morbidity. ${ }^{2,4,5}$ Fiber dissections and neuroimaging techniques, such as MRI tractography, are increasingly used to supplement anatomical knowledge by showing the relationship between the lesion and the eloquent white matter tracts; this assists surgeons with the selection of an approach ${ }^{7,8}$ and decreases morbidity associated with manipulation of the brainstem during resection. In cases of lesions located deep in the ventral midbrain, the anterior mesencephalic (i.e., perioculomotor) safe entry zone has been described by Cavalcanti et al. ${ }^{2}$ and Kalani et al. ${ }^{4}$ This safe entry zone makes use of the placement of the pyramidal tract, which is located in the middle threefifths of the cerebral peduncle, to remove ventral lesions by entering the brainstem medial to this tract, lateral to the oculomotor nerve (cranial nerve [CN] III), and between the posterior cerebral artery and the superior cerebellar artery. However, no safe entry zone has been described to reach deep lesions located ventral and medial to the oculomotor nerve in the midbrain. In this study, we describe the interpeduncular fossa as a novel, safe entry zone in the ventral midbrain for resection of deep lesions located medial to the oculomotor nerve. We illustrate the utility of this approach with 2 clinical cases of patients treated for cavernous malformations, and we supplement these examples with anatomically pertinent measurements of cadaveric dissections to define this new safe entry zone.

\section{Methods}

Six formalin-fixed adult human brainstems and 4 adult human cadaveric heads were injected with colored silicone and examined. The human brainstems were dissect-

ABBREVIATIONS $\mathrm{CN}=$ cranial nerve.

SUBMITTED June 27, 2016. ACCEPTED September 22, 2016.

INCLUDE WHEN CITING Published online March 10, 2017; DOI: 10.3171/2016.9.JNS161680.

* Drs. Kalani and Yağmurlu contributed equally to this work. 
ed using fiber dissection techniques with microdissectors under magnifications of $6 \times$ to $40 \times$ with a Zeiss Surgical Microscope (Carl Zeiss AG). An orbitozygomatic entry technique was used on the heads to approach the interpeduncular safe entry zone. Quantitative measurements were performed to obtain the relevant surgical parameters.

The clinical and radiographic histories of a 59-yearold male patient and a 28 -year-old female patient were reviewed retrospectively to document neurological examination and radiographic parameters.

\section{Results}

\section{Surgical Anatomy of the Interpeduncular Fossa}

The interpeduncular fossa has a wedge-shaped depression between the cerebral peduncles and contains the posterior perforated substance in its floor ${ }^{6}$ (Figs. 1 and 2). The perforating arteries pass through the posterior perforated substance to supply the thalamus. The peduncular vein arises in the interpeduncular fossa and courses laterally around the cerebral peduncle toward the basal vein. The oculomotor nerve exits from the lateral walls of the fossa, and oculomotor rootlets form the lateral wall of the interpeduncular fossa. The interpeduncular cistern is located between the cerebral peduncles. The apex of the basilar artery usually sits inside the interpeduncular cistern and in front of the interpeduncular fossa. The interpeduncular cisterns extend laterally as the crural cisterns.

Quantitative anatomical measurements were performed to define the pertinent surgical parameters. The mean depth of the surgical trajectory, meaning the distance from the craniotomy edge to the interpeduncular fossa measured $8.2 \pm 0.6 \mathrm{~cm}( \pm \mathrm{SD})$. The mean distance between the optic nerve (CN II) and the internal carotid artery was $0.4 \pm 0.05 \mathrm{~cm}$. The mean distance between the optic nerve and the oculomotor nerve was $0.9 \pm 0.2 \mathrm{~cm}$, and the mean distance between the oculomotor nerve and the internal carotid artery was $0.5 \pm 0.1 \mathrm{~cm}$. The basilar

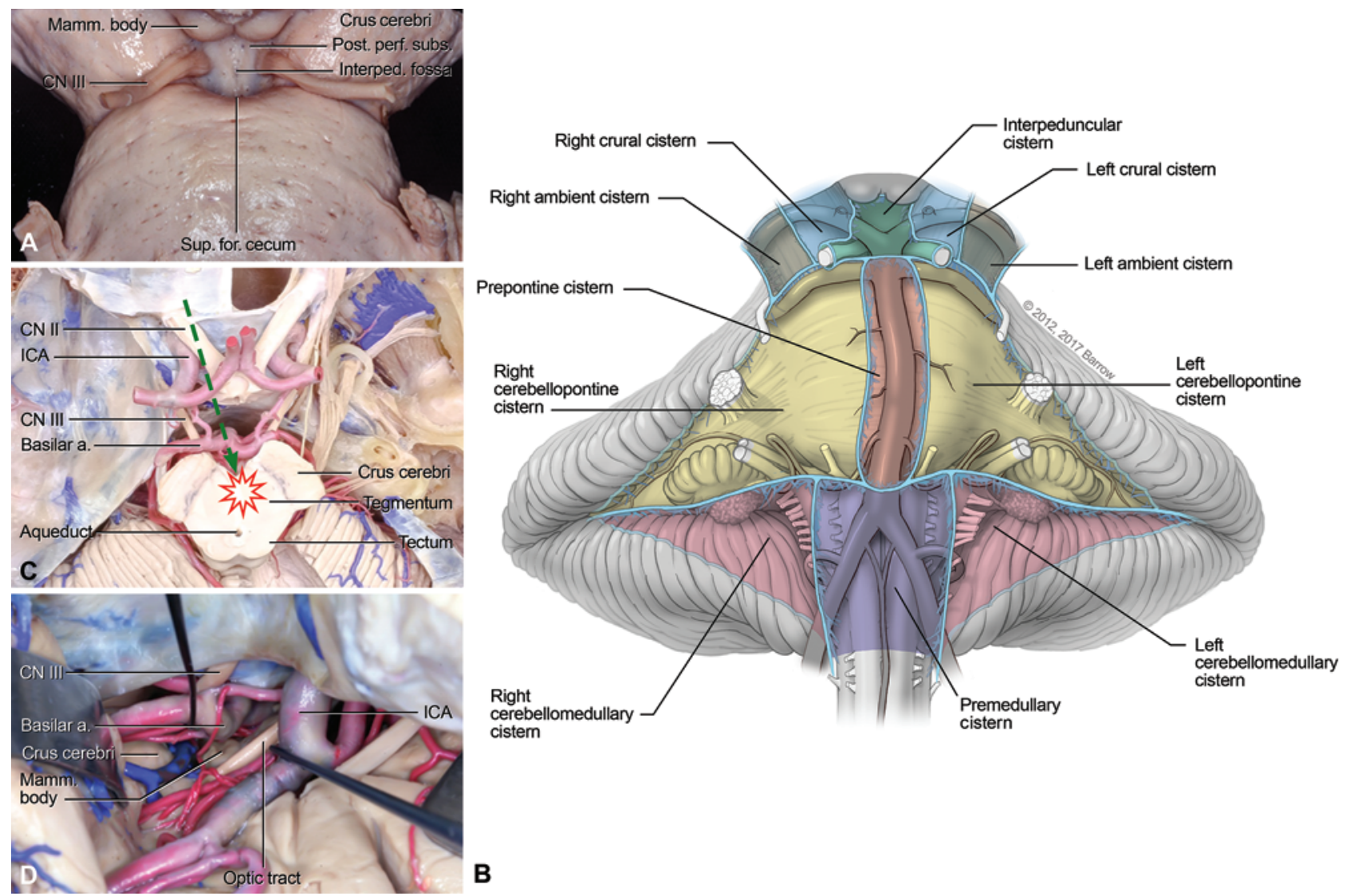

FIG. 1. Descriptive anatomy of the region of interest. A: The interpeduncular fossa has a wedge-shaped depression between the cerebral peduncles and contains the posterior perforated substance in its floor. The most caudal point of the fossa is the superior foramen cecum, which is a depression in the midline. The oculomotor nerves (CN III) exit from the lateral walls of the fossa. B: The basilar apex sits in front of the interpeduncular fossa and gives rise to the perforator arteries, which feed the posterior perforated substance in the fossa wall. C: Superior view of the approach trajectory (dashed arrow) to a lesion (starburst) located in the tegmentum of the midbrain. An entry through the interpeduncular fossa reaches the tegmentum of the midbrain. D: Left view of the surgical route showing the passage between the basilar apex and mammillary body to reach the interpeduncular fossa. a. = artery; $\mathrm{CN}$ = cranial nerve; for. = foramen; $\mathrm{ICA}$ = internal carotid artery; Interped. = interpeduncular; Mamm. = mammillary; perf. $=$ perforated; Post. = posterior; subs. = substance; Sup. = superior. Panels A, C, and D: dissections prepared by Kaan Yağmurlu, MD. Reproduced with permission from the Rhoton Collection. (http://rhoton.ineurodb.org), CC BY-NC-SA 4.0 (http:// creativecommons.org/licenses/by-nc-sa/4.0). Panel B: Copyright Barrow Neurological Institute, Phoenix, Arizona. Published with permission. Figure is available in color online only. 

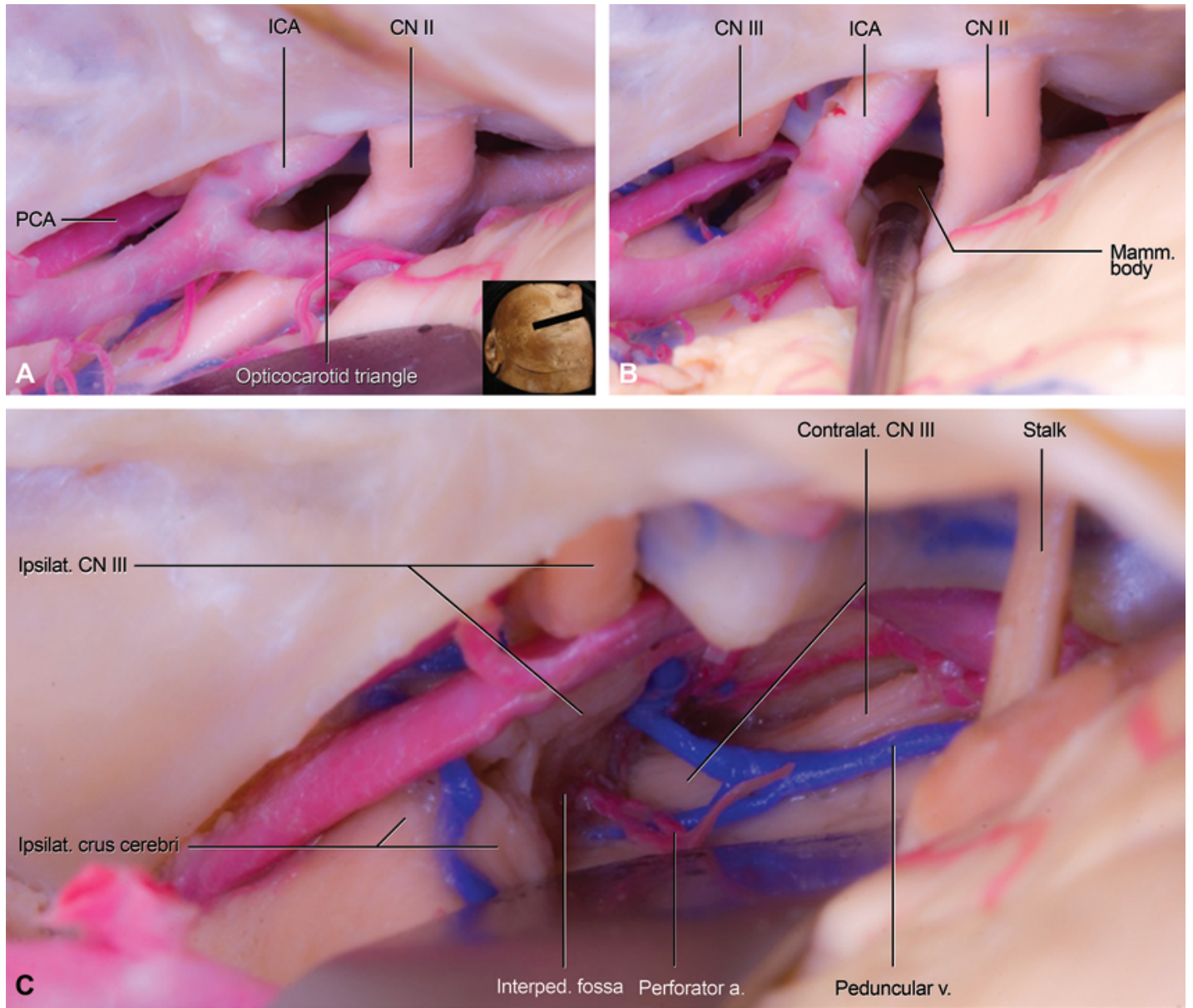

FIG. 2. Surgical steps. A: After the left modified orbitozygomatic craniotomy is performed, the opticocarotid complex is exposed. The inset shows head orientation. B: The dissection proceeds by passing through the opticocarotid triangle. Thereafter, the mammillary body and basilar apex can be identified. (In this specimen, the basilar apex is seated very low.) C: Further dissection exposes the bilateral oculomotor nerves, interpeduncular fossa, and basilar perforating arteries. Contralat. = contralateral; Ipsilat. = ipsilateral; PCA = posterior cerebral artery; v. = vein. Dissections prepared by Kaan Yağmurlu, MD. Reproduced with permission from the Rhoton Collection. (http://rhoton.ineurodb.org), CC BY-NC-SA 4.0 (http://creativecommons.org/licenses/by-nc-sa/4.0). Figure is available in color online only.

perforators that arise from the terminal part of the vessel ranged from 1 to 5 and most commonly arose from the posterior surface of the basilar artery.

\section{Case Reports Case 1}

A 59-year-old right-handed man was transferred to our clinical service after sudden-onset slurring of speech and right hemiplegia. The patient was intubated and had flickering (i.e., minimally flexing in response to painful stimulus) in his right upper and lower extremities. In contrast, he was able to briskly withdraw his left upper and lower extremities. MRI revealed a large hematoma and an associated cavernous malformation located in the midbrain. The lesion most closely approached a pial surface on the ventral surface of the brainstem (Fig. 3A-C).

The patient was taken to the operative suite for imageguided resection of the lesion using a right-sided modified orbitozygomatic approach. Given the location of the lesion, the lateral displacement of the basilar artery, and the fact that the lesion most closely approximated a pial surface at the level of the interpeduncular fossa, we elected to approach the lesion using the interpeduncular fossa safe entry zone. We worked between the optic nerve and the carotid artery to arrive at the interpeduncular fossa, ultimately developing the space between the mammillary bodies and the top of the basilar artery to incise the midbrain (Fig. 3D-F). Although we monitored somatosensory evoked potentials, motor evoked potentials, and specific cranial nerves, we did not perform direct stimulation in this case. The lesion was resected in a gross-total fashion (Fig. 3G and H). Postoperatively, the patient experienced improvement of his symptoms and was able to follow commands for the left side (4/5) and to flex the right upper and lower extremities. He received a tracheostomy and was transitioned to a skilled nursing facility.

\section{Case 2}

A 28-year-old right-handed woman was referred to our vascular service with a history of sudden-onset diplopia 

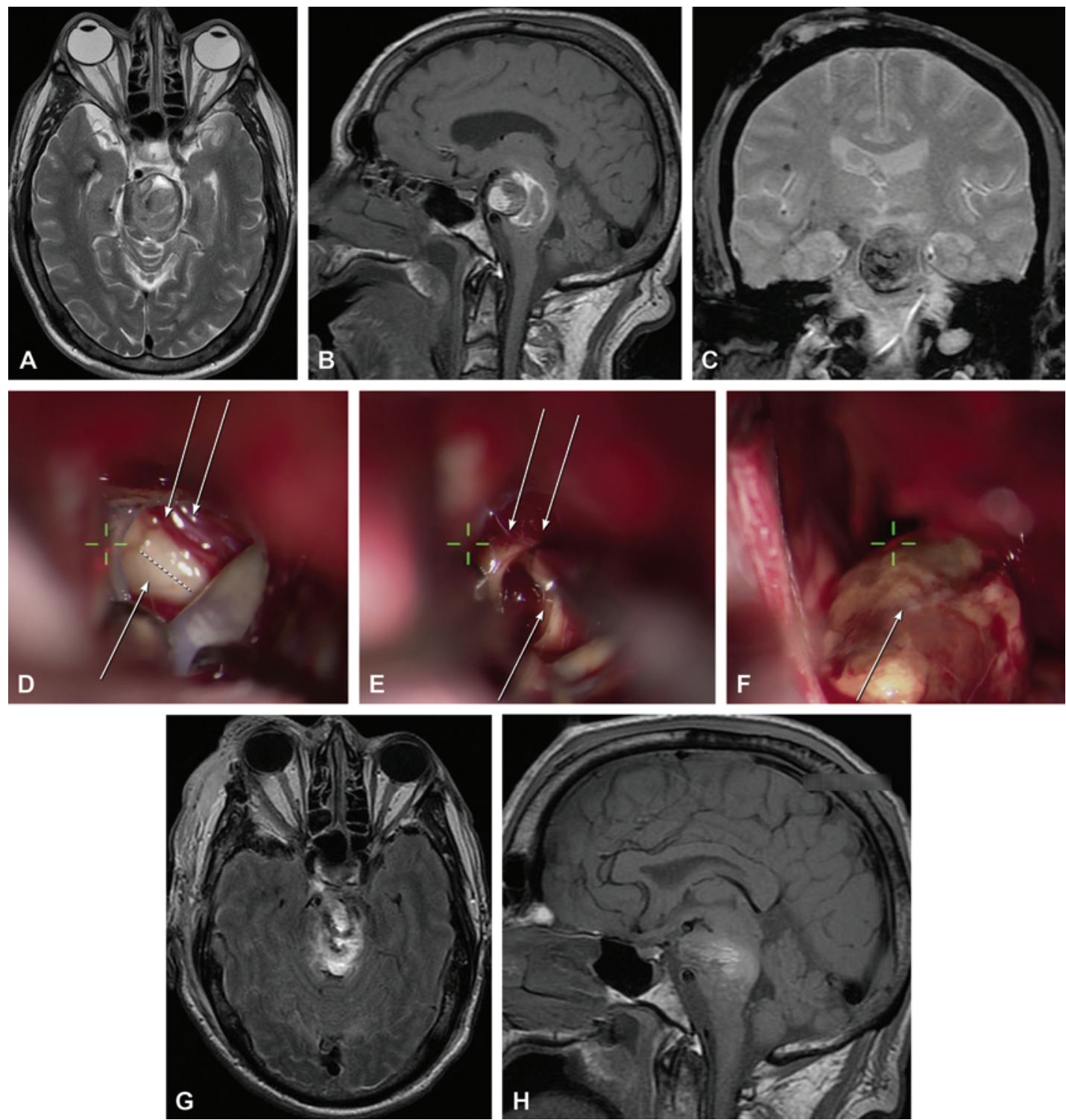

FIG. 3. Case 1. A 59-year-old right-handed man presented with acute-onset slurring of speech and right hemiplegia. A-C:

Preoperative axial T2-weighted (A), sagittal T1-weighted (B), and coronal gradient echo (C) MRI sequences reveal a large mesencephalopontine hemorrhage caused by rupture of a cavernous malformation. Note that the lesion most closely approximates a pial surface on the ventral surface of the midbrain. The basilar artery is displaced laterally to the right side. Via a right-sided modified orbitozygomatic craniotomy, the space between the carotid artery and the optic nerve is opened to arrive at the interpeduncular fossa safe entry zone. This tight corridor is accessed by dissecting the space between the mammillary bodies and the top of the basilar artery. D: The surgeon carefully mobilizes the perforating arteries at the top of the basilar artery (arrows) to incise the brainstem (dotted line). E: The brainstem is entered, taking care not to disturb the basilar perforators (arrows). F: The lesion (arrow) is then mobilized and removed in a gross-total fashion. G and H: Postoperative axial T2-weighted (G) and sagittal T1weighted $(\mathrm{H}) \mathrm{MR}$ images demonstrating complete removal of the lesion. Copyright Barrow Neurological Institute, Phoenix, Arizona. Published with permission. Figure is available in color online only.

and right upper- and lower-extremity weakness that had completely resolved by the time of presentation. MRI revealed a deep cavernous malformation located in the left midbrain and medial to the oculomotor nerve. The lesion closely approached a pial surface on the ventral surface of the brainstem and at the level of the interpeduncular fossa (Fig. 4A-C).

The lesion was approached with a right-sided (contralateral) modified orbitozygomatic craniotomy using neuronavigation to enter the brainstem via the interpeduncular fossa safe entry zone. The decision to approach this lesion, located in the left midbrain, with a right-sided craniotomy was dictated by the medial location of the lesion relative to the oculomotor nerve, which precluded the use of the anterior mesencephalic (i.e., perioculomotor) safe entry zone. The lesion was resected in a gross-total fashion (Fig. 4D and E). Postoperatively, the patient's status remained unchanged. 

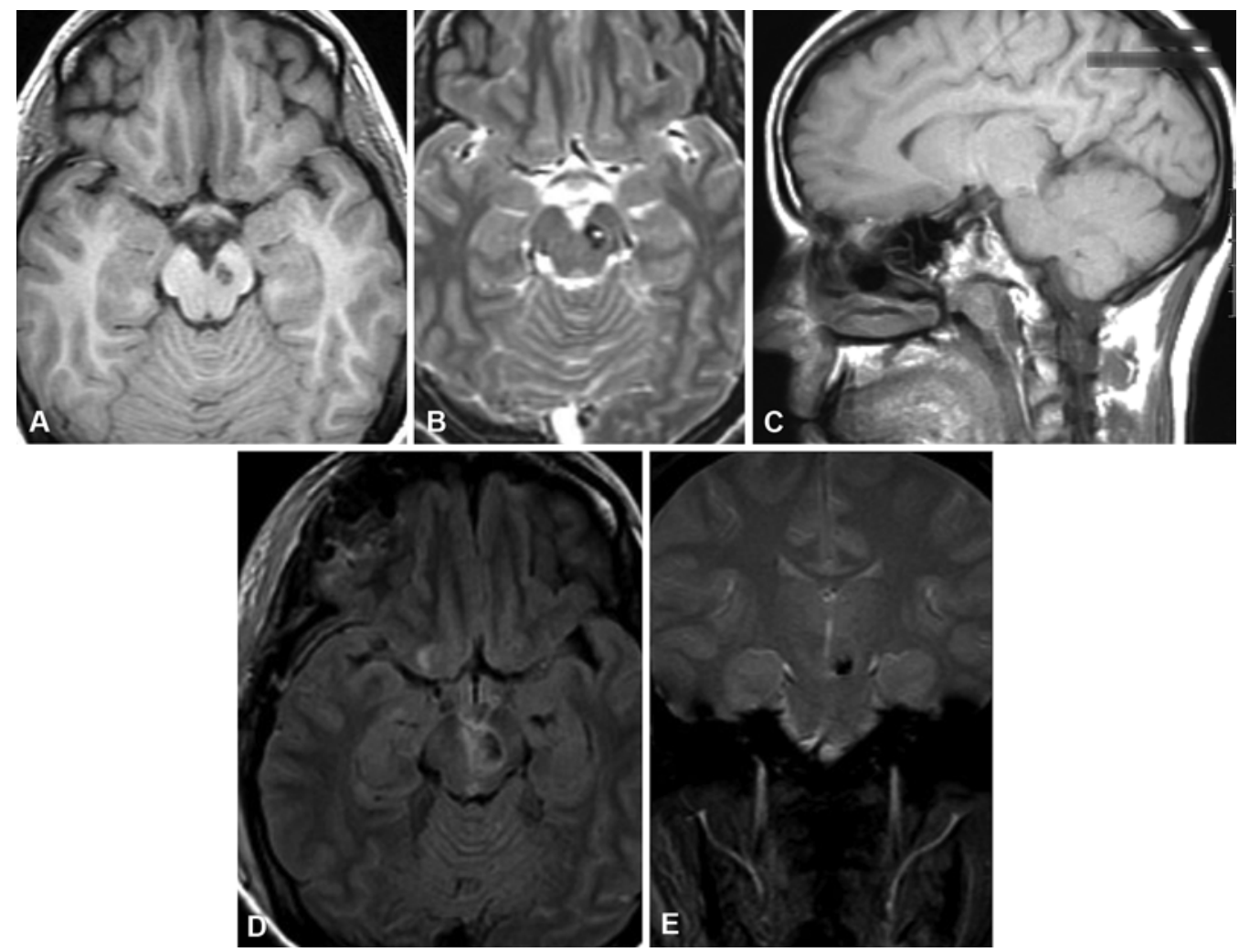

FIG. 4. Case 2. A 28-year-old right-handed woman was evaluated for a history of sudden onset diplopia and right upper- and lower-extremity weakness. Preoperative axial T1-weighted (A), axial T2-weighted (B), and sagittal T1-weighted (C) MR images demonstrating a cavernous malformation located in the left ventral midbrain. The lesion is located medial to the oculomotor nerve on the left side and most closely approximates a pial surface at the interpeduncular fossa. The lesion was approached using a contralateral (right-sided) modified orbitozygomatic approach. The surgeon worked between the optic nerve (CN II) and the internal carotid artery to arrive at the interpeduncular fossa. A working corridor was developed by working between the mammillary bodies and the perforators arising from the top of the basilar artery, and the midbrain was incised to completely remove the lesion. Postoperative axial T1-weighted (D) and coronal gradient echo (E) MRI sequences demonstrate gross-total removal of the cavernous malformation.

\section{Discussion}

When lesions reach the surface of the brainstem, they often create a natural corridor for their removal. In these cases, approach selection is dictated by the 2-point method. ${ }^{1}$ For deep-seated lesions, the use of the 2-point method in conjunction with described safe entry zones allows the surgeon to minimize injury to critical structures., 4 The anterior mesencephalic (i.e., perioculomotor) entry zone is an accepted safe entry zone for lesions located in the ventral midbrain. ${ }^{4}$ This safe entry zone makes use of the location of the pyramidal tract to gain access to the ventral midbrain; the pyramidal tract is located in the middle three-fifths of the cerebral peduncle and lateral to the exit point of the oculomotor nerve in the interpeduncular fossa. The anterior mesencephalic (i.e., perioculomotor) safe entry zone is located lateral to the oculomotor nerve exit from the brainstem, medial to the pyramidal tract, and is bound rostrocaudally by the posterior cerebral artery and the superior cerebellar artery. Lesions located medial to the oculomotor nerve are often not accessible via this safe entry zone, and a lateral or posterolateral approach may be more appropriate for resection of these lesions. For example, the lateral supracerebellar infratentorial approach can be used to resect lateral or dorsolateral midbrain lesions, often via the lateral mesencephalic sulcus safe entry zone as previously described. ${ }^{3,4,5}$ For lesions located in the ventromedial midbrain and medial to the oculomotor nerve, the interpeduncular safe entry zone can be used to remove lesions with acceptable patient morbidity.

The key to using the interpeduncular fossa safe entry zone is to remain flexible and at times to resort to using a contralateral approach with a modified orbitozygomatic craniotomy to obtain the trajectory and view necessary to remove lesions from the ventromedial midbrain. As demonstrated by the quantitative anatomical measurements, the working room afforded by this approach is minute, and care must be taken to use multiple operative corridors to visualize and resect lesions in this confined space. Although the oculomotor nerve is not well visualized in the operative photographs of the case illustrations, it does serve as the lateral limit of the interpeduncular cistern and should be identified prior to entry into the brainstem. In approaching the interpeduncular fossa, the surgeon begins by using the working window between the optic nerve (CN II) and the carotid artery to arrive at the ventral surface of the midbrain. This area affords little workspace and is bound superiorly by the mammillary body and infe- 
riorly by the basilar artery apex and the perforators arising from the basilar artery. Care must be taken to avoid injury when mobilizing the basilar perforating arteries, as the loss of even a single vessel can result in devastating ischemic complications for the patient. Laterally, the limiting structures are the posterior cerebral arteries and the oculomotor nerve. When incising the brainstem, the surgeon should place the incision in the direction of the ascending/ descending or crossing fibers in the area of interest. The interpeduncular fossa is free of descending motor fibers, and crossing fibers are scarce, making this area ideal for entry into the brainstem. We prefer a rostrocaudal incision in the interpeduncular fossa that is parallel to the basilar perforators and allows their minimal manipulation.

The 2-point method is used to determine the safest and shortest route from the pial-ependymal surface of the brainstem to deep lesions. ${ }^{1}$ Whenever possible, the safe entry zones should be combined with the 2-point method to identify the ideal surgical route for deep-seated lesions. As in the cases presented in this report, for lesions located in the ventral midbrain, medial to the oculomotor nerve and not abutting a pial surface, the ideal approach is an orbitozygomatic craniotomy and transinterpeduncular entry into the brainstem. It is important to note that a contralateral approach may be necessary at times.

\section{Conclusions}

The interpeduncular fossa may be used as a safe entry zone for ventromedial lesions located in the mesencephalon. The use of the orbitozygomatic craniotomy allows the surgeon to obtain the necessary trajectory and view to develop the space between the mammillary bodies and the basilar apex to incise the midline anterior midbrain and remove lesions from this area with acceptable morbidity.

\section{Acknowledgments}

We acknowledge the Neuroscience Publications office at Barrow Neurological Institute for assistance with preparation of this manuscript. All dissections and photography were performed by the second author (K.Y.) at the Dr. Albert L. Rhoton Neuro-Microanatomy Lab at the University of Florida, Gainesville, Florida, and the Neuroanatomy Laboratory at Barrow Neurological Institute in Phoenix, Arizona.

\section{References}

1. Brown AP, Thompson BG, Spetzler RF: The two-point method: Evaluating brain stem lesions. BNI Q 12:20-24, 1996

2. Cavalcanti DD, Preul MC, Kalani MYS, Spetzler RF: Microsurgical anatomy of safe entry zones to the brainstem. J Neurosurg 124:1359-1376, 2016

3. Kalani MYS, Martirosyan NL, Nakaji P, Spetzler RF: The supracerebellar infratentorial approach to the dorsal midbrain. Neurosurg Focus 40 (Video Suppl 1):15462, 2016

4. Kalani MYS, Yagmurlu K, Martirosyan NL, Cavalcanti DD, Spetzler RF: Approach selection for intrinsic brainstem pathologies. J Neurosurg 125:1596-1607, 2016

5. Kalani MYS, Yagmurlu K, Martirosyan NL, Spetzler RF: The retrosigmoid petrosal fissure transpeduncular approach to central pontine lesions. World Neurosurg 87:235-241, 2016

6. Rhoton AL Jr: Cerebellum and fourth ventricle. Neurosurgery 47 (3 Suppl):S7-S27, 2000

7. Ulrich NH, Kockro RA, Bellut D, Amaxopoulou C, Bozinov $\mathrm{O}$, Burkhardt JK, et al: Brainstem cavernoma surgery with the support of pre- and postoperative diffusion tensor imaging: initial experiences and clinical course of 23 patients. Neurosurg Rev 37:481-492, 2014

8. Yagmurlu K, Rhoton AL Jr, Tanriover N, Bennett JA: Three-dimensional microsurgical anatomy and the safe entry zones of the brainstem. Neurosurgery 10 (Suppl 4):602-620, 2014

\section{Disclosures}

The authors report no conflict of interest concerning the materials or methods used in this study or the findings specified in this paper.

\section{Author Contributions}

Conception and design: all authors. Acquisition of data: Kalani, Yağmurlu. Analysis and interpretation of data: Kalani, Yağmurlu. Drafting the article: Kalani, Yağmurlu. Critically revising the article: all authors. Reviewed submitted version of manuscript: Spetzler, Kalani. Study supervision: Spetzler.

\section{Correspondence}

Robert F. Spetzler, c/o Neuroscience Publications, Barrow Neurological Institute, St. Joseph's Hospital and Medical Center, 350 W Thomas Rd., Phoenix, AZ 85013. email: neuropub@dignityhealth. org. 